\title{
Detection of the Presence of Extended-Spectrum Beta- Lactamase Enzyme in Gram-negative Bacteria According to CLSI and EUCAST Criteria
}

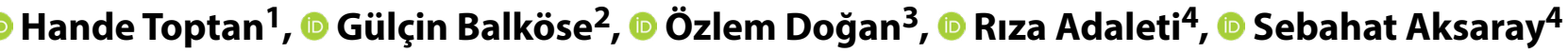 \\ ${ }^{1}$ Department of Medical Microbiology, Sakarya University, Sakarya, Turkey \\ ${ }^{2}$ Department of Medical Microbiology, Mugla Sitki Kocman Training and Research Hospital, Mugla, Turkey \\ ${ }^{3}$ Department of Medical Microbiology, Koc University, Istanbul, Turkey \\ ${ }^{4}$ Department of Medical Microbiology, University of Health Sciences Turkey, Haydarpasa Numune Training and Research Hospital, \\ Istanbul, Turkey
}

\begin{abstract}
Introduction: Expanded spectrum beta-lactamase is a critical source that is limiting the using areas of beta-lactam antibiotics by hydrolysing them. The method to be used for accurate detection of ESBL production is significant. CLSI and EUCAST are the two most commonly used standards for the determination of antibiotic susceptibility. This study aimed to investigate the sensitivity and specificity of these standards for ESBL detection.

Methods: This study includes 76 ESBL producers and 74 ESBL negative strains, which were isolated from urine specimens that sent to the Medical Microbiology Laboratory of Haydarpasa Numune Training and Research Hospital from April to July 2014. For screening tests, ceftazidime $(10 \mu \mathrm{g}$ and $30 \mu \mathrm{g})$, cefotaxime $(5 \mu \mathrm{g}$ and $30 \mu \mathrm{g})$, ceftriaxone $(30 \mu \mathrm{g})$, cefpodoxime $(10 \mu \mathrm{g})$, cefepime $(30 \mu \mathrm{g})$ ve aztreonam $(30 \mu \mathrm{g})$ disks; for confirmation tests double-disk synergy and combination disk method were used. For molecular confirmation tests, CTX-M, TEM and SHV resistance genes were investigated by polymerase chain reaction.

Results: ESBL producing isolates were found at $86.8 \%$ as CTX-M, 47.4\% as TEM and $9.2 \%$ as SHV. More than one resistance gene was detected in some isolates. Beta-lactam susceptibilities of ESBL producers with CLSI and EUCAST breakpoints were $29.2 \%$ and $19.7 \%$ for ceftazidime, $14.5 \%$ and $15.8 \%$ for cefepime, $2.6 \%$ and $13.2 \%$ for aztreonam, respectively. Among the screening disks, cefpodoxime (interpreted by EUCAST criteria), showed the highest sensitivity and specificity. This was followed by cefpodoxime (CLSI), cefotaxime and ceftriaxone with similar sensitivity and specificity for both standards.

Discussion and Conclusion: There was no significant difference between CLSI and EUCAST in determining ESBL production. The suggestion of EUCAST that using different disks for screening and confirmation tests increases the sensitivity of detecting ESBL, especially in ceftazidime; however, it may cause practical difficulties. For both standards, the use of cefpodoxime, cefotaxime or ceftriaxone disks in screening and; cefotaxime and cefotaxime- clavulanate disks in confirmation tests will provide more accurate results.

Keywords: Confirmatory test; CLSI; Expanded spectrum beta-lactamase; EUCAST; screening test.
\end{abstract}

$E^{x}$ xpanded-spectrum beta-lactamases are increasing in number and prevalence, and are a significant source of resistance to hydrolysis of beta-lactam antibiotics. Expand- ed-spectrum beta-lactamases are microbiologically defined as enzymes that can hydrolyze oxyimino cephalosporins and which can be inhibited by clavulanic acid ${ }^{[1]}$. 
ESBL-producing bacteria may produce outbreaks in hospitals by transferring these enzymes among species ${ }^{[2]}$. Since ESBLs are able to inactivate beta-lactam antibiotics other than carbapenems by hydrolysis, clinicians face severe problems in the treatment of Gram-negative bacteria, especially Klebsiella spp. and Escherichia coli. In addition, ESBL-producing strains have multiple antibiotic resistances due to the transport of other resistance genes with the same plasmids as the genes responsible for the production of ESBL ${ }^{[3]}$. The presence of these enzymes is also associated with high morbidity and mortality rates, especially in bacteremia. Because of the reasons, such as resistance to many antibiotics, prolongation of hospital stay, increased morbidity and mortality rates and severe economic losses, it should be known whether the isolate constitutes the ESBL enzyme or not ${ }^{[4]}$.

Because of the need for accurate detection of ESBL production, the method to be used for ESBL detection becomes important. Antimicrobial susceptibility in all over the world is performed according to certain standards and most commonly used are the Clinical and Laboratory Standards Institute (CLSI) and the European Committee on Antimicrobial Susceptibility Testing (EUCAST). In our country, CLSI standards have been used for many years in the evaluation and application of antimicrobial susceptibility tests (AST). However, for reasons such as EUCAST allowing free access to all its documents, our resistance profiles and susceptibility patterns may be closer to European isolates due to our geographical proximity and being able to provide standardized data for international surveillance studies, EUCAST standards have been adopted in microbiology laboratories.

EUCAST and CLSI have some discrepancies concerning some disc contents, media to be used, sensitivity ranges for some combinations of bacteria and antibiotics. One of these differences is the content and sensitivity limit values of discs used in the investigation of ESBL production of bacteria belonging to the family of Enterobacterales ${ }^{[5,6]}$. This study aims to evaluate the characteristics of the Enterobacterales family by detecting the properties of ESBL production by comparing the CLSI and EUCAST standards with the molecular method.

Based on the idea that this situation may cause significant differences in the results report, in our study, it was aimed to evaluate the ability of CLSI and EUCAST to detect ESBL production in bacteria of the Enterobacterales family by adopting the molecular method as the gold standard.

\section{Materials and Methods}

This study was approved by the Haydarpaşa Numune Training and Research Hospital Ethical Committee, Protocol no: 24.02.2016 version: 1.
From April 2014 to July 2014, 150 isolates from the Enterobacterales family, isolated from urine samples taken from patients in various clinics and sent to Haydarpasa Numune Training and Research Hospital Medical Microbiology Laboratory were included in this study. Seventy-six isolates suspected of ESBL positivity with resistance to at least one of the 3rd generation cephalosporins were included as study group and 74 isolates, which are sensitive to all $3^{\text {rd }}$ generation cephalosporins and considered to be ESBL negative were included as a control group in this study.

All of the isolates included in this study were tested for screening and phenotypic confirmatory tests (combination disc and double-disk synergy methods) by disk diffusion method and genotypic confirmatory tests.

The antibiotic discs to be studied for susceptibility testing were selected from the ESBL screening discs recommended by either CLSI or EUCAST guidelines. After the isolate to be tested was adjusted to 0.5 Mcfarland turbidity, the suspension was spread to three Mueller Hinton media. Ceftazidime $(30 \mu \mathrm{g})$, ceftazidime $(10 \mu \mathrm{g})$, cefotaxime $(30 \mu \mathrm{g})$, cefotaxime $(5 \mu \mathrm{g})$, aztreonam $(30 \mu \mathrm{g})$, cefpodoxime $(10 \mu \mathrm{g})$, ceftriaxone $(30 \mu \mathrm{g})$, cefepime $(30 \mu \mathrm{g})$ discs were placed for screening tests. For the double-disk synergy test, screening disks were placed at a distance of $20 \mathrm{~mm}$ from the center of amoxicillin-clavulanate $(20+10 \mu \mathrm{g})$ disk. For combination disk method ceftazidime- clavulanate $(30+10 \mu \mathrm{g})$ and cefotaxime- clavulanate $(30+10 \mu \mathrm{g})$ disks were used (Fig. 1). After 18-20 hours incubation, the diameters of the inhibition zones formed around the discs were measured in millimeters using a ruler, and the results were interpreted separately according to the breakpoints of CLSI and EUCAST $(2017)^{[5,6]}$.

For DNA extraction, the isolates were passaged to MuellerHinton agar medium. After 24 hours incubation, the colonies were suspended in 0.5 McFarland turbidity in $200 \mu \mathrm{l}$ Tris-Acetate-EDTA (TAE) buffer. Foam tube rack supported suspension was then inserted in a tube, which was supported by a foam tube rack and boiled in a flask filled

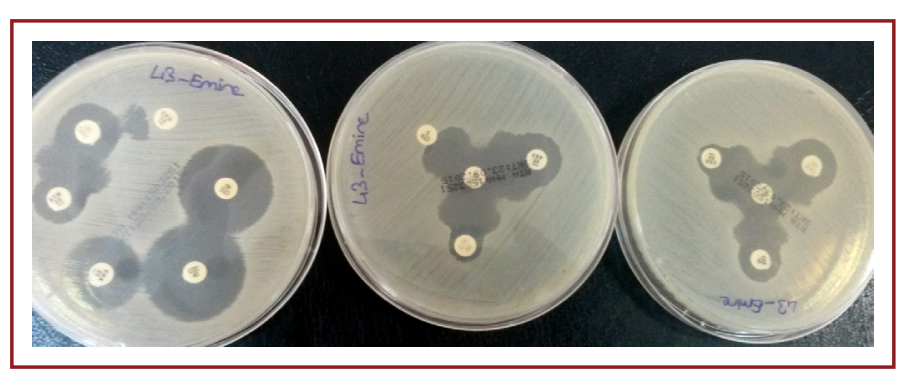

Figure 1. Antibiogram image of ESBL-producing E.coli isolate at the end of 18 hours of incubation. 
with water for 10 minutes at $100{ }^{\circ} \mathrm{C}$. The supernatant obtained after centrifugation at $13000 \mathrm{rpm}$ for 10 minutes was used as template DNA for amplification.

Using the forward and reverse primers for the TEM, SHV and CTX-M gene regions for each isolate (Table 1), the PCR protocol was performed in a thermal cycler (Techne, England) as: 10 min first denaturation at $94^{\circ} \mathrm{C}$; a total of 30 cycles of 1 min denaturation at $94{ }^{\circ} \mathrm{C}, 1 \mathrm{~min}$ annealing at $58 / 62{ }^{\circ} \mathrm{C}$, and $1 \mathrm{~min}$ elongation at $72^{\circ} \mathrm{C}$; and $10 \mathrm{~min}$ final elongation at $72{ }^{\circ} \mathrm{C}$. The amplified products were imaged by agarose gel electrophoresis (Fig. 2).

For statistical analysis, NCSS (Number Cruncher Statistical System) 2007 (Kaysville, Utah, USA) program was used. Descriptive statistical methods (mean, standard deviation, median, frequency, ratio, minimum, maximum) were used to evaluate the study data. McNemar and Kappa compliance tests and diagnostic screening (e.g., specificity and sensitivity) tests were used for the compatibility of the CLSI

\begin{tabular}{ccc}
\multicolumn{2}{c}{ Table 1. Primers used and expected amplicon sizes ${ }^{[12]}$} & \\
\hline Targeted Gene Region & Primer alignment (5'-3') & Size (bp) \\
\hline TEM & & \\
F & TCCGCTCATGAGACAATAACC & 931 \\
R & TTGGTCTGACAGTTACCAATGC & \\
SHV & & \\
F & TGGTTATGCGTTATATTCGCC & 868 \\
R & GGTTAGCGTTGCCAGTGCT & \\
CTX-M & & \\
F & & \\
R & TCTTCCAGAATAAGGAATCCC & 909 \\
\hline
\end{tabular}

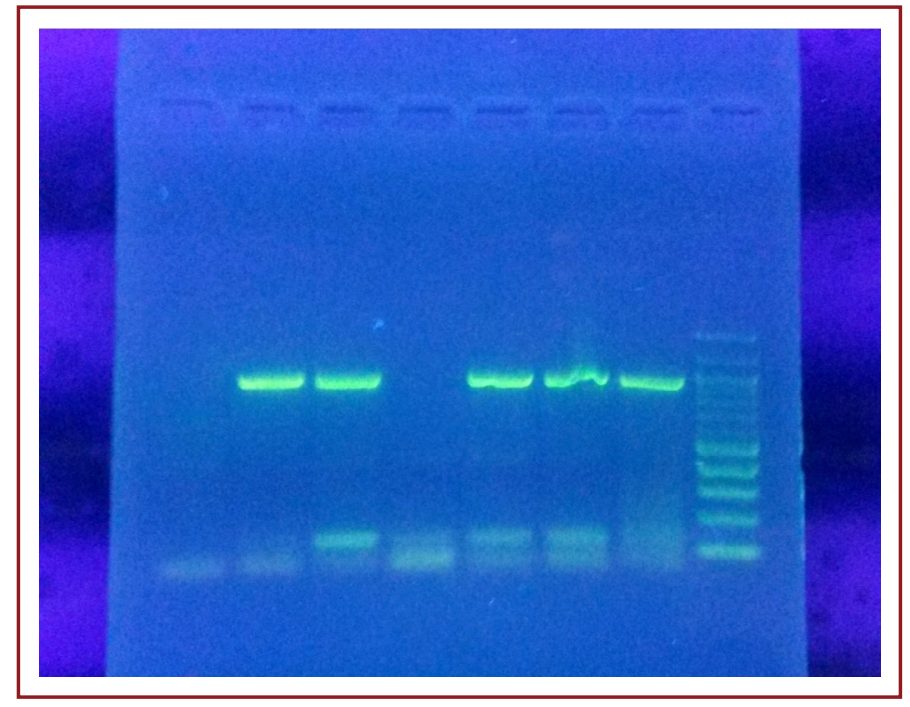

Figure 2. Image of the bands on a transilluminator at the end of the electrophoresis. and EUCAST criteria with each other and with the molecular method.

\section{Results}

The ages of the cases ranged from 18 to 93 years, with an average of $50.87 \pm 21.19$ years. $23.3 \%$ of the cases were male and $76.7 \%$ were female. E.coli was isolated from $80 \%$ of the cases and ESBL was most commonly found in K. pneumoniae $(85 \%)$ and especially in intensive care samples $(71.4 \%)$ (Table 2). In our study, $86.8 \%$ of ESBL producing isolates were found as CTX-M, $47.4 \%$ were TEM and $9.2 \%$ were SHV. More than one resistance gene was detected in some isolates. Beta lactam susceptibilities of ESBL producing isolates were determined as $29.2 \%$ and $19.7 \%$ for ceftazidime, $14.5 \%$ and $15.8 \%$ for cefepime, $2.6 \%$ and $13.2 \%$ for aztreonam, using CLSI and EUCAST breakpoints respectively. Among the screening disks, cefpodoxime disk (interpreted by the EUCAST criteria), showed the highest sensitivity and specificity. This was followed by cefpodoxime disk interpreted by CLSI criteria and by cefotaxime and ceftriaxone disks with similar sensitivity and specificity for both criteria. Ceftazidime was the lowest sensitive screening disc, according to both CLSI and EUCAST criteria (Table 3).

In the double-disk synergy test, synergy was found between cefotaxime (both CLSI and EUCAST discs), cefpodoxime and ceftriaxone discs and amoxicillin clavulanate discs in all isolates. This rate was $89.5 \%$ for ceftazidime, 98.7\% for cefepim and $97.4 \%$ for aztreonam. In combination methods, this rate was $\mathbf{7 7 . 6 \%}$ for ceftazidime + ceftazidime/clavulanate; and $98.7 \%$ for cefotaxime + cefotaxime/clavulanate.

Table 2. Types of bacterial species and their distribution by clinics

\begin{tabular}{|c|c|c|c|c|}
\hline & \multicolumn{2}{|c|}{$\begin{array}{c}\text { Total } \\
(n=150)\end{array}$} & \multicolumn{2}{|c|}{$\begin{array}{l}\text { ESBL producers } \\
\qquad(\mathbf{n = 7 6 )}\end{array}$} \\
\hline & $\mathbf{n}$ & $\%$ & $\mathbf{n}$ & $\%$ \\
\hline \multicolumn{5}{|l|}{ Bacterial species } \\
\hline E.cloacae & 2 & 1.3 & 1 & 50 \\
\hline E.coli & 120 & 80 & 56 & 46.7 \\
\hline K.pneumoniae & 20 & 13.4 & 17 & 85 \\
\hline P.mirabilis & 8 & 5.3 & 2 & 25 \\
\hline \multicolumn{5}{|l|}{ Clinic } \\
\hline Emergency room & 16 & 10.6 & 5 & 31.3 \\
\hline $\mathrm{ICU}$ & 7 & 4.7 & 5 & 71.4 \\
\hline Surgical medicine & 19 & 12.7 & 7 & 36.8 \\
\hline İnternal medicine & 40 & 26.7 & 24 & 60 \\
\hline Urology & 58 & 38.7 & 30 & 51.7 \\
\hline Others & 10 & 6.7 & 5 & 50 \\
\hline
\end{tabular}


Table 3. Sensitivity and specificity of screening discs according to CLSI and EUCAST breakpoints

\begin{tabular}{|c|c|c|c|c|c|c|c|c|c|c|c|c|}
\hline & $\begin{array}{l}\text { FEP* } \\
\text { CLSI }\end{array}$ & $\begin{array}{c}\text { FEP } \\
\text { EUCAST }\end{array}$ & $\begin{array}{c}\text { CRO* } \\
\text { CLSI }\end{array}$ & $\begin{array}{c}\text { CRO } \\
\text { EUCAST }\end{array}$ & $\begin{array}{c}\text { CAZ* } \\
\text { CLSI }\end{array}$ & $\begin{array}{c}\text { CAZ } \\
\text { EUCAST }\end{array}$ & $\begin{array}{l}\text { CTX* } \\
\text { CLSI }\end{array}$ & $\begin{array}{c}\text { CTX } \\
\text { EUCAST }\end{array}$ & $\begin{array}{c}\text { CPD* } \\
\text { CLSI }\end{array}$ & $\begin{array}{c}\text { CPD } \\
\text { EUCAST }\end{array}$ & $\begin{array}{c}\text { ATM* } \\
\text { CLSI }\end{array}$ & $\begin{array}{c}\text { ATM } \\
\text { EUCAST }\end{array}$ \\
\hline Sensitivity & 85.5 & 84.2 & 98.7 & 97.4 & 61.8 & 80.3 & 98.7 & 98.7 & 98.7 & 100 & 97.4 & 86.8 \\
\hline Specificity & 100 & 100 & 89.2 & 98.7 & 100 & 97.3 & 94.6 & 98.7 & 98.7 & 100 & 94.6 & 100 \\
\hline $\mathrm{PPV}^{* *}$ & 100 & 100 & 90.4 & 98.7 & 100 & 96.8 & 94.9 & 98.7 & 98.7 & 100 & 94.9 & 100 \\
\hline NPV*** & 87.1 & 86.1 & 98.5 & 97.3 & 71.8 & 82.8 & 98.6 & 98.7 & 98.7 & 100 & 97.2 & 88.1 \\
\hline
\end{tabular}

${ }^{*}$ FEP: Cefepime; CRO: Ceftriaxone; CAZ: Ceftazidime; CTX: Cefotaxime; CPD: Cefpodoxime; ATM: Aztreonam; **PPV: Positive predictive value; ${ }^{* * *}$ NPV: Negative predictive value.

\section{Discussion}

Many studies have been conducted to compare the breakpoints of the two committees in the determination of ESBL production after 2010. Blandino et al. ${ }^{[7]}$ reported in their study in 2014 that they found all 200 ESBL producing isolates as resistant to cefepime, ceftazidime, cefotaxime and aztreonam using EUCAST and CLSI breakpoints. RodriguezBano et al. ${ }^{[8]}$ reported that ceftazidime sensitivity was 58.6\% (CLSI) and 14.7\% (EUCAST) and cefepim sensitivity as $35.1 \%$ (CLSI) and $14.7 \%$ (EUCAST) in their study with 191 ESBL-producing E. coli. Hombach et al. ${ }^{[9]}$ found that 150 ESBL-producing isolates belonging to Enterobacterales family were susceptible to ceftazidime and cefepime in $38.7 \%$ and $28 \%, 58.7 \%$ and $24 \%$, respectively, when CLSI and EUCAST breakpoints were applied, the results for cefpodoxime, cefotaxime and ceftriaxone were similar. In that study, EUCAST breakpoints were one step ahead when ceftazidime and cefepime were used. Our findings suggest that EUCAST breakpoints for ceftazidime and CLSI breakpoints for aztreonam was more sensitive in detecting ESBL. This may be because EUCAST does not include aztreonam screening breakpoint, in our study, we used routine clinical breakpoint for aztreonam.

Pollfuss et al. ${ }^{[10]}$ performed a study that identified the sensitivity and specificity of each agent using 118 of ESBL-producing 236 Enterobacterales isolates using cephalosporin screening breakpoints of CLSI and EUCAST. According to this study, cefpodoxime (EUCAST) was the agent with the highest sensitivity, while the agent with the highest specificity was cefepime, which was evaluated using CLSI breakpoints. They attributed this to the presence of both ESBL and Amp C producing isolates. In our study, only cefoxitin sensitive isolates were included, so we think that Amp C masking was partially avoided. In our study, the highest sensitivity and specificity were observed in cefpodoxime disc, which is interpreted according to EUCAST breakpoints.
Other good sensitivity and specificity balanced discs were cefpodoxime (CLSI), cefotaxime and ceftriaxone, respectively. Despite its high specificity, ceftazidime is considered to be the most unsuccessful agent due to its low sensitivity. In this study, we think that there is a large number of betalactamase type CTX-M and this type of beta-lactamases are relatively ineffective on ceftazidime.

EUCAST recommends $10 \mu \mathrm{g}$ disk for ceftazidim, $5 \mu \mathrm{g}$ disc for cefotaxime in screening tests and $30 \mu \mathrm{g}$ discs for both agents in combination disc diffusion test. This suggestion is believed to increase the ESBL detection rate, but we think that in daily practice, the need for different discs may cause some difficulties. In our study, the sensitivity of cefotaxime screening test was found to be the same in accordance with the recommendations of both committees, whereas ceftazidime sensitivity was found to be higher than that of CLSI when the EUCAST breakpoints were used.

In our study, ESBL production of isolates was also investigated by the molecular method as a gold standard method to reliably compare screening and confirmation tests. Thus, we investigated the presence of gene regions responsible for the most common extended-spectrum beta-lactamase production by PCR. Although the ranking may vary, the most frequent ESBL resistance gene regions in Turkey and in the world are CTX-M, SHV and TEM ${ }^{[11]}$. Hombach et al. ${ }^{[9]}$ found that in 150 ESBL-producing isolates, $92 \%$ were CTX-M, $7.3 \%$ were SHV and $0.7 \%$ was TEM. Polsfuss et al. ${ }^{[10]}$ found the CTX-M, SHV and TEM gene regions in 118 isolates at $89.8 \%, 9.3 \%$ and $1.7 \%$, respectively. In Turkey, Görgeç et al. ${ }^{[12]}$ conducted a study with 76 ESBL-producing E.coli in Malatya in 2015 and they found CTX-M, TEM and SHV gene regions $89.5 \%, 59.2 \%$ and $11.8 \%$, respectively. In our study, $86.8 \%$ of ESBL-producing isolates were CTX-M, $47.4 \%$ were TEM and $9.2 \%$ were SHV.

Our study has a few limitations since it has included only one hospital data and has a limited number of strains. In 
this study, cefoxitin sensitivity was used to prevent AmpC's masking of ESBL detection and cefoxitin-resistant isolates were excluded ${ }^{[13]}$. In the presence of AmpC type betalactamase false positivity in screening tests with an ESBL negative isolate or false-negative results in the confirmation tests of an ESBL-producing isolate may be detected, resulting in unnecessary labor, time and cost loss. To prevent these losses, we believe that it would be beneficial to develop our study with tests that will make the separation of these two types of beta-lactamase more safely. In our study, the sensitivity for screening and confirmation tests was consistent with similar studies in the literature, but their specificity was found to be quite high. The reason for this is that the ESBL negative group has included isolates that have very low MICs for cephalosporin group antibiotics. Working with isolates having a MIC closer to the CLSI or EUCAST susceptibility limit of the respective antibiotic will result in a more balanced sensitivity and specificity. In our study, although we have focused on AST and molecular typing due to budget limitations in ESBL detection, we are aware that this issue should not be considered separately from carbapenem resistance. We think that with continuation of the study by increasing the number of strains and detecting also the carbapenem resistance, we think that this dark spot can be enlightened on this issue where many clinical microbiology labs are indecisive.

As a result, there was no significant difference between the CLSI and EUCAST breakpoints in the detection of ESBL production. EUCAST's suggestion on different contents for ceftazidim and cefotaxim discs for screening and verification disk diffusion tests may increase susceptibility especially for ceftazidime; but we believe that this may lead to some the practical use difficulties. For both standards using cefpodoxime, cefotaxime or ceftriaxone discs in screening tests and using of cefotaxime and cefotaximeclavulonate discs for confirmation tests will provide more accurate results.

Ethics Committee Approval: Haydarpaşa Numune Training and Research Hospital Ethical Committee, Protocol no: 24.02.2016 version: 1.

Peer-review: Externally peer-reviewed.

Authorship Contributions: Concept: H.T., S.A.; Design: H.T., R.A.; Data Collection or Processing: H.T., G.B.; Analysis or Interpretation: H.T., O.D.; Literature Search: H.T., G.B.; Writing: H.T.

Conflict of Interest: None declared.

Financial Disclosure: The authors declared that this study received no financial support.

\section{References}

1. Rawat D, Nair D. Extended-spectrum $\beta$-lactamases in Gram Negative Bacteria. J Glob Infect Dis 2010;2:263-74. [CrossRef]

2. Dhillon RH, Clark J. ESBLs: A Clear and Present Danger? Crit Care Res Pract 2012;2012:625170. [CrossRef]

3. Taşova Y. Gram negatif enterik bakteri enfeksiyonlarının yönetimi, ANKEM Derg 2011;25:34-44.

4. de Kraker ME, Wolkewitz M, Davey PG, Koller W, Berger J, Nagler J, et al. Burden of antimicrobial resistance in European hospitals: excess mortality and length of hospital stay associated with bloodstream infections due to Escherichia coli resistant to third-generation cephalosporins. J Antimicrob Chemother 2011;66:398-407. [CrossRef]

5. CLSI. M100 Performance Standards for Antimicrobial Susceptibility Testing. 27th ed. Wayne, PA: Clinical and Laboratory Standards Institute; 2017.

6. EUCAST. Breakpoint Tables for Interpretation of MICs and Zone Diameters, Version 7.1, 2017. Available at: http://www. eucast.org/ast_of_bacteria/previous_versions_of_documents/. Accessed 20 Nov, 2018.

7. Blandino G, Mastrojeni S, Inturri R, Sciacca A, Nicoletti G. Antimicrobial susceptibility of strains of Enterobactericeae isolated from bloodstream infections using current CLSI and EUCAST breakpoints. Health 2014;6:153-7. [CrossRef]

8. Rodríguez-Baño J, Picón E, Navarro MD, López-Cerero L, Pascual A; ESBL-REIPI Group. Impact of changes in CLSI and EUCAST breakpoints for susceptibility in bloodstream infections due to extended-spectrum $\beta$-lactamase-producing Escherichia coli. Clin Microbiol Infect 2012;18:894-900. [CrossRef]

9. Hombach M, Mouttet B, Bloemberg GV. Consequences of revised CLSI and EUCAST guidelines for antibiotic susceptibility patterns of ESBL- and AmpC $\beta$-lactamase-producing clinical Enterobacteriaceae isolates. J Antimicrob Chemother 2013;68:2092-8. [CrossRef]

10. Polsfuss S, Bloemberg GV, Giger J, Meyer V, Hombach M. Comparison of European Committee on Antimicrobial Susceptibility Testing (EUCAST) and CLSI screening parameters for the detection of extended-spectrum $\beta$-lactamase production in clinical Enterobacteriaceae isolates. J Antimicrob Chemother 2012;67:159-66. [CrossRef]

11. Coque TM, Baquero F, Canton R. Increasing prevalence of ESBL-producing Enterobacteriaceae in Europe. Euro Surveill 2008;13:19044.

12. Görgeç S, Kuzucu Ç, Otlu B, Yetkin F, Ersoy Y. Genişlemiş spektrumlu beta-laktamaz üreten nozokomiyal Escherichia coli izolatlarında beta-laktamaz gen oranları ve klonal ilişkinin araştırılması [Investigation of beta-lactamase genes and clonal relationship among the extended-spectrum betalactamase producing nosocomial Escherichia coli isolates]. Mikrobiyol Bul 2015;49:15-25. [CrossRef]

13. Philippon A, Arlet G, Jacoby GA. Plasmid-determined AmpC-type beta-lactamases. Antimicrob Agents Chemother 2002;46:1-11. [CrossRef] 